\title{
Reversible structural transition in nanoconfined ice
}

\author{
V. Satarifard, ${ }^{1}$ M. Mousaei, ${ }^{1,2}$ F. Hadadi,,${ }^{1,3}$ James Dix, ${ }^{4}$ M. Sobrino Fernandez, ${ }^{5}$ P. Carbone, ${ }^{4}$ J. Beheshtian,,${ }^{1,6}$ \\ F. M. Peeters, ${ }^{5}$ and M. Neek-Amal ${ }^{1,5,6, *}$ \\ ${ }^{1}$ Institute for Advanced Technologies, Shahid Rajaee Teacher Training University, Lavizan, Tehran 16785-136, Iran \\ ${ }^{2}$ Department of Physics and Mechanical Engineering, Sharif University of Technology, P.O. Box 11365-9567, Tehran, Iran \\ ${ }^{3}$ Department of Physics, Shahid Beheshti University, G.C. Evin, Iran \\ ${ }^{4}$ School of Chemical Engineering and Analytical Science, The University of Manchester, Manchester M13 9PL, United Kingdom \\ ${ }^{5}$ Department of Physics, Universiteit Antwerpen, Groenenborgerlaan 171, B-2020 Antwerpen, Belgium \\ ${ }^{6}$ Department of Physics, Shahid Rajaee Teacher Training University, Lavizan, Tehran 16785-136, Iran \\ (Received 31 August 2016; revised manuscript received 14 December 2016; published 15 February 2017)
}

\begin{abstract}
The report on square ice sandwiched between two graphene layers by Algara-Siller et al. [Nature (London) $\mathbf{5 1 9}, 443$ (2015)] has generated a large interest in this system. By applying high lateral pressure on nanoconfined water, we found that monolayer ice is transformed to bilayer ice when the two graphene layers are separated by $\mathrm{H}=6,7 \AA$. It was also found that three layers of a denser phase of ice with smaller lattice constant are formed if we start from bilayer ice and apply a lateral pressure of about $0.7 \mathrm{GPa}$ with $\mathrm{H}=8,9 \AA$. The lattice constant (2.5-2.6 $\AA$ ) in both transitions is found to be smaller than those typical for the known phases of ice and water, i.e., $2.8 \AA$. We validate these results using $a b$ initio calculations and find good agreement between ab initio O-O distance and those obtained from classical molecular dynamics simulations. The reversibility of the mentioned transitions is confirmed by decompressing the systems.
\end{abstract}

DOI: 10.1103/PhysRevB.95.064105

\section{INTRODUCTION}

Graphene, the two-dimensional allotrope of carbon [1], has been used recently to confine water into two-dimensional layers [2]. Algara-Siller et al. claimed that they detected monolayer, bilayer, and trilayer ice using transmission electron microscopy (TEM). The water molecules were found to be ordered into a square lattice. The lateral pressure for confining water between two sheets of graphene can be estimated to be 1 GPa using the van der Waals (vdW) adhesive energy between two layers, which is typically around $20 \mathrm{meV}^{-2}$ [2-4]. Molecular dynamics (MD) simulations, using a nanocapillary setup, predicted that by increasing pressure up to $1 \mathrm{GPa}(1.35$ $\mathrm{GPa}$ ), bilayer square ice (trilayer ice) with a lattice constant of $2.83 \AA$ is formed between two graphene layers that are separated by $\mathrm{H}=9 \AA$ (11.5 $\mathrm{\AA}$ ), which was found to be in agreement with electron energy loss spectra [2]. The confinement width needed for the formation of a stable monolayer of ice was found to be $\mathrm{H} \simeq 5-7 \AA[2,5,6]$. Later, Zhou et al. argued that the above-mentioned experiment can be better explained as due to salt (for example, $\mathrm{NaCl}$ ) contaminants precipitating as nanocrystals between the graphene layers [7].

Notwithstanding the existing MD-based theoretical studies of the past few years, there are still challenging questions, such as how important the boundary condition is in common MD simulations and its consequence on the structural properties of nanoconfined water [8-10]. Most of the water phases, e.g., liquid water, ice structures, and confined ice, have a lattice constant around $2.8 \AA$. Therefore, the next question is as follows: Can we find a dense ice phase with smaller lattice constant using common simulation methods? One naturally expects that by increasing the lateral pressure, the $\mathrm{O}-\mathrm{O}$ distance becomes shorter, resulting in a denser structure for confined ice.

\footnotetext{
*neekamal@srttue.du
}

Here we show that the particular boundary conditions will change the structural properties of confined water. To clarify this, we use two different MD classical force fields (i.e., TIP4P(2005) [11] and ReaxFF [12]) and ab initio calculations. The ReaxFF potential is used to estimate the H-bonding energy and to show that by increasing pressure, independent of the number of layers, the H-bonding energy decreases nonlinearly. We found reasonable agreement between $a b$ initio and MD force-field calculations for the $\mathrm{O}-\mathrm{O}$ distance between the water molecules (2.45-2.6 $\AA$ ). The reversibility of the monolayer ice to bilayer and the bilayer ice to trilayer transitions is confirmed by expanding and decompressing the system. We also studied the effects of channel size.

\section{METHOD AND MODEL}

\section{A. Model}

Our system is comprised of three elements (see Fig. 1): (i) two rigid graphene layers (with dimensions $10 \times 10 \mathrm{~nm}^{2}$ ) which are fixed at $\mathrm{H}=8,9 \AA(6,7 \AA)$ distance. The graphene layers are in $x-z$ plane and are considered for studying the transition from bilayer (monolayer) to trilayer (bilayer), (ii) two walls for controlling the lateral pressure, and (iii) $N_{W}$ water molecules which are randomly distributed inside the chamber $\left(N_{W}\right.$ varies between 1000 and 1800 depending on the chosen $\mathrm{H}$ ). Our setup is different from the one used by Algara-Silleret et al. [2] in that we use two rigid walls for applying pressure. The dimension of the moving wall is 10 $\mathrm{nm} \times \mathrm{H}$. We employed molecular dynamics (MD) simulations in the well-known large-scale atomic/molecular massively parallel simulator (LAMMPS) [13]. We kept the temperature fixed at $298 \mathrm{~K}$ using Nosé-Hoover thermostats as implemented in LAMMPS. Before starting the compression, we relaxed the system for $1 \mathrm{~ns}$ and found monolayer and bilayer ice for systems with $\mathrm{H}=6$ and $9 \AA$, respectively. The obtained structures for bilayer ice after the first equilibration step 


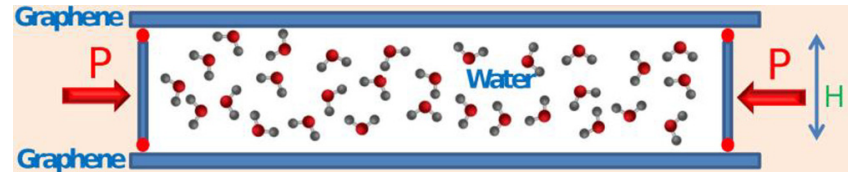

FIG. 1. Schematic setup of confined water between two graphene layers held at a distance $\mathrm{H}$ from each other.

were $\mathrm{AB}$ stacking, relevant to the previous reports [2]. For long-range interactions, we use a cutoff of $10 \AA$.

Since the external pressure in a real experiment can be controlled, e.g., by injecting gas around the system, the lateral walls move, resulting in a compression confined water yielding bilayer (monolayer) ice when $\mathrm{H}=8,9 \AA$ (6,7 $\AA$; see Fig. 1$)$. By moving the walls further or, equivalently, by increasing the pressure beyond $6 \mathrm{GPa}$, trilayer ice (or bilayer) is formed (see Fig. 2). The used walls can be considered as a model for the observed "terraces" with sharp steps in graphene in the real experiment [2]. In our calculations, periodic boundary conditions are applied in the $y$ direction.

\section{B. TIP4P model}

To model the water-water interaction, we used the four-site interaction potential TIP4P(2005). In TIP4P(2005) [11], the electrostatic interaction is modeled by using Coulomb's law and the dispersion and repulsion forces by using the LennardJones (LJ) potential. The nonbonded potential energy between the water molecules in the TIP4P model is given by

$$
E_{n b}=\sum_{i, j \neq i} \frac{k q_{i} q_{j}}{r_{i j}}+\sum_{i, j \neq i} \sum_{m=1,2} 4 \epsilon(-1)^{m}\left(\frac{\sigma}{r_{i j}}\right)^{6 m},
$$

where $k=332.1 \AA \mathrm{kcal} / \mathrm{mol}$, and $q_{i}$ and $q_{j}$ are the partial charges over the $i$ th and $j$ th atoms which are separated by $r_{i j}$. In Eq. (1), $\sigma$ and $\epsilon$ are commonly used LJ parameters which are set to $\epsilon_{O O}=0.185 \mathrm{kcal} / \mathrm{mol}, \sigma_{O O}=3.1589 \AA$, and $\epsilon_{C C}=0.056 \mathrm{kcal} / \mathrm{mol}, \sigma_{C C}=3.4 \AA$ for TIP4P(2005). Force-field parameters of carbon and water interaction are adopted from Ref. [14]. Though Eq. (1) is a very simple model to simulate water, it has been shown that it captures most of the physics of water and many studies have been published that explain various physical properties of water using the aforementioned classical models [15-17]. In the TIP4P(2005) model, the charge over the $\mathrm{O}$ atoms and $\mathrm{M}$ sites is -1.1128 e and on the $\mathrm{H}$ atoms is $0.5564 e$, which is fixed during the simulations. The distance between the $\mathrm{O}$ and $\mathrm{M}$ site is 0.1564 $\AA$, where $\mathrm{M}$ is the dummy atom in the TIP4P model.

Notice that in the original paper of TIP4P(2005) [11], there are many test simulations for the validation of this force field, including phase diagram, equation of state at high pressure, and melting of water. The calculated properties include a number of thermodynamic properties of the solid and liquid phases, as well as several other properties of the liquid. From the point of view of the thermodynamic conditions, the results of Ref. [11] covered a temperature range from 123 to $573 \mathrm{~K}$, and pressures up to $4 \mathrm{GPa}$. Therefore, this force field is expected to be suitable for our purposes where GPa pressures are applied on water. (a)
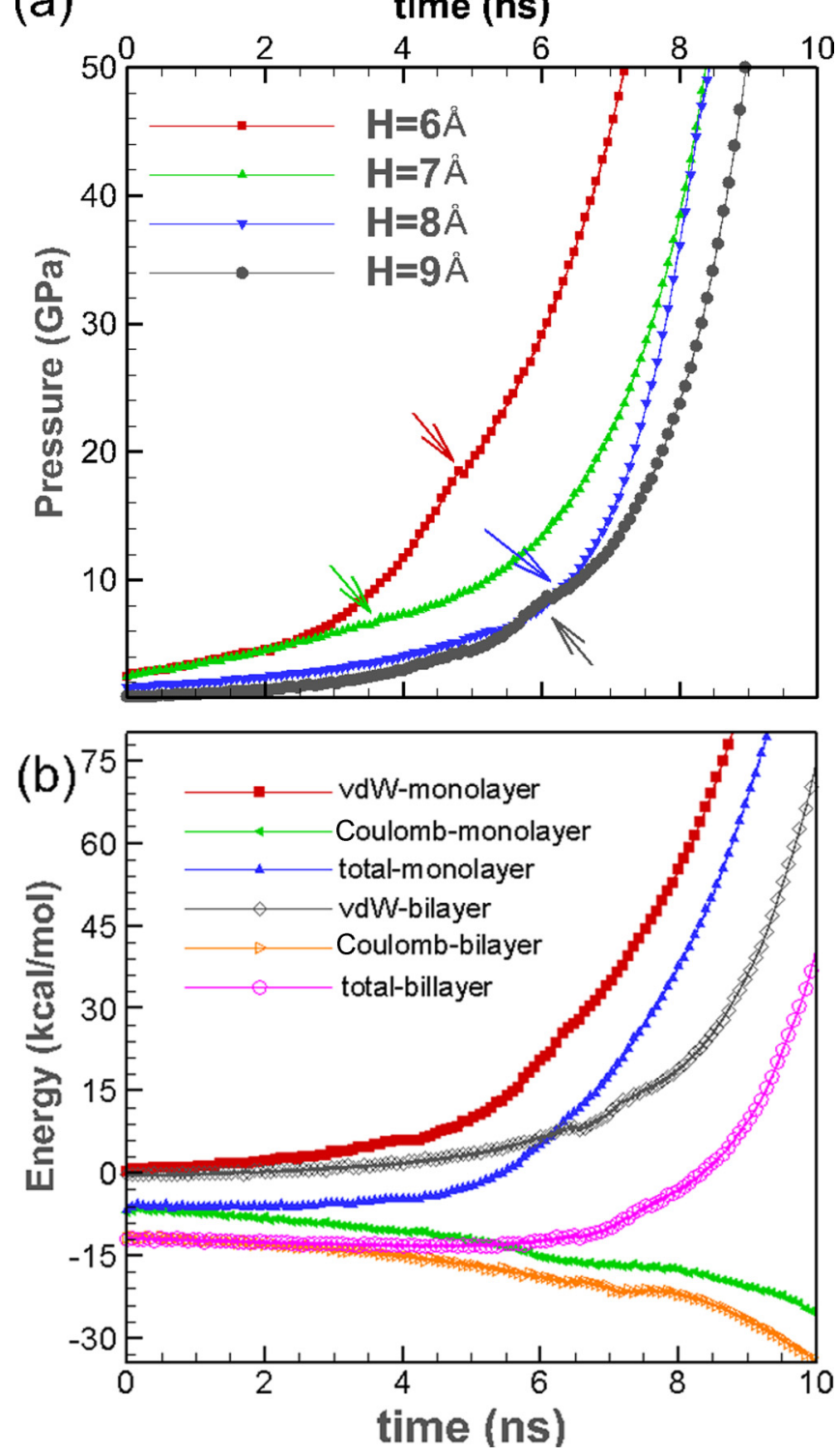

FIG. 2. (a) The variation of calculated pressure with MD simulation time in nanoconfined water, which is initially monolayer (bilayer) ice and ended at bilayer (trilayer) ice. The legends refer to different channel sizes. (b) The different energy contributions (i.e., $\mathrm{vdW}$, Coulomb, and total energy) of nanoconfined water as a function of lateral pressure for monolayer (bilayer) ice transformed to bilayer (trilayer) ice calculated using TIP4P. Here $\mathrm{H}=6,9 \AA$ for monolayer (bilayer) to bilayer (trilayer) transitions, respectively.

\section{Reactive force-field potential}

In order to provide an independent check on the results, we also used the reactive force field (ReaxFF) that allows for a larger flexibility of the interaction potential $[9,10,18]$. The reactive force field divides the system energy up into various partial energy contributions, such as the self-energy which captures the difference in energy of the various charge states of an atom, the Coulomb term, which captures the electrostatic interactions, the terms for nonbonded interactions through the van der Waals energy, and the bond-order terms which are corrected for overcoordination to allow for the long-range 


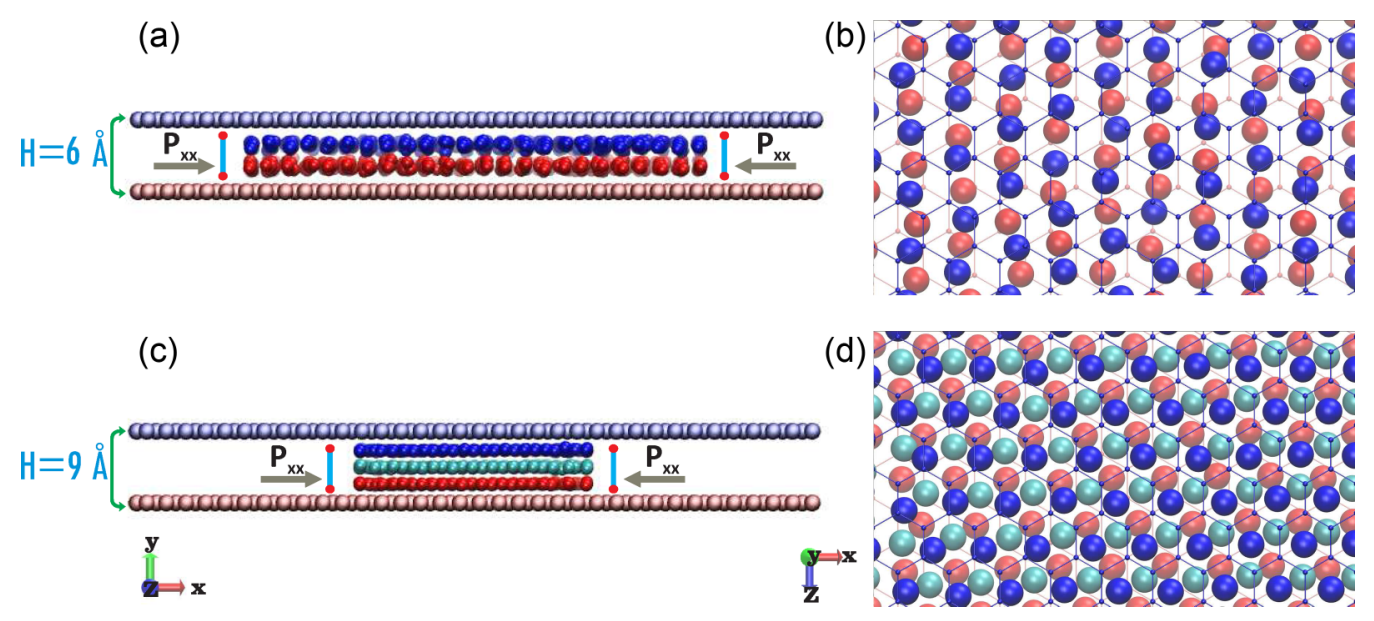

FIG. 3. (a) Side and (b) top view of a snapshot of our molecular dynamics simulation for bilayer ice at $T=298 \mathrm{~K}$. We obtain trilayer ice at about $0.7 \mathrm{GPa}$ where we show a (c) side and (d) top view. We show only the oxygen atoms, where different colors refer to the different layers.

bond order necessary for accurate reaction barriers. ReaxFF also explicitly includes energy contributions related to valence and torsion angle distortion energy contribution related to three-body and four-body conjugation, a weak hydrogen bond term to properly describe $X-\mathrm{H}-X$ nonbonded interactions in polar molecules, energy terms related to the number of valence electrons around an atom, etc. [12].

One of the main advantages of ReaxFF is that it calculates the polarization of charge within the molecules, which is achieved by using electronegativity and hardness parameters based on the electronegativity equalization method and charge equilibration $(\mathrm{QEq})$ methods. Furthermore, the ReaxFF potential allows bond extension/contraction in water as well as angle bending, and it allows charge relaxation over each atom. In fact, the electrostatic energy is minimized with the equilibrium charge distribution under the total charge constraints. Charges are updated at every step, which is the most computational part of the ReaxFF energy calculations. In this study, we use ReaxFF to find H-bonding energy that is estimated to be in good agreement with DFT results, i.e., -0.11 , $-0.16 \mathrm{eV} /$ water $[9,10,19]$.

\section{D. $A b$ initio model}

All of our density functional theory (DFT) calculations were carried out within the generalized gradient approximations (GGA) for the exchange-correlation energy term using QUANTUM ESPRESSO [20]. The double numerical plus polarization (DNP) basis set and Perdew-Burke-Ernzerhof (PBE) functional [21] were adopted. Because the weak interactions are not well described by the standard PBE functional, we adopted a PBE-D approach (D stands for dispersion) with the Grimme vdW correction [22]. This approach is a hybrid semiempirical solution that introduces damped atom-pairwise dispersion correction of $\mathrm{C}^{6} \mathrm{R}^{-6}$ form in the DFT formalism. The accuracy of the DNP basis set is comparable to that of the $6-31 \mathrm{G}^{* *}$ basis set. The Brillouin-zone integration was performed using $4 \times 4 \times 2$ Monkhorst-Pack $k$-point sampling [23]. The structure optimization was symmetry unrestricted and was carried out using the conjugate-gradient algorithm. The convergence criterion of our self-consistent calculations for energy is chosen as $10^{-5} \mathrm{eV}$ between two consecutive steps. We use periodic boundary conditions within the supercell geometry, and the vacuum spacing between graphene layers in adjacent supercells is $25 \AA$. For partial occupancies, the Gaussian smearing method is used. We distributed the water molecules between the graphene layers randomly with four different initial configurations.

\section{RESULTS}

\section{A. MD simulations}

We start to compress water between two graphene layers, which are separated by distance of 8 and $9 \AA$ (in the case of

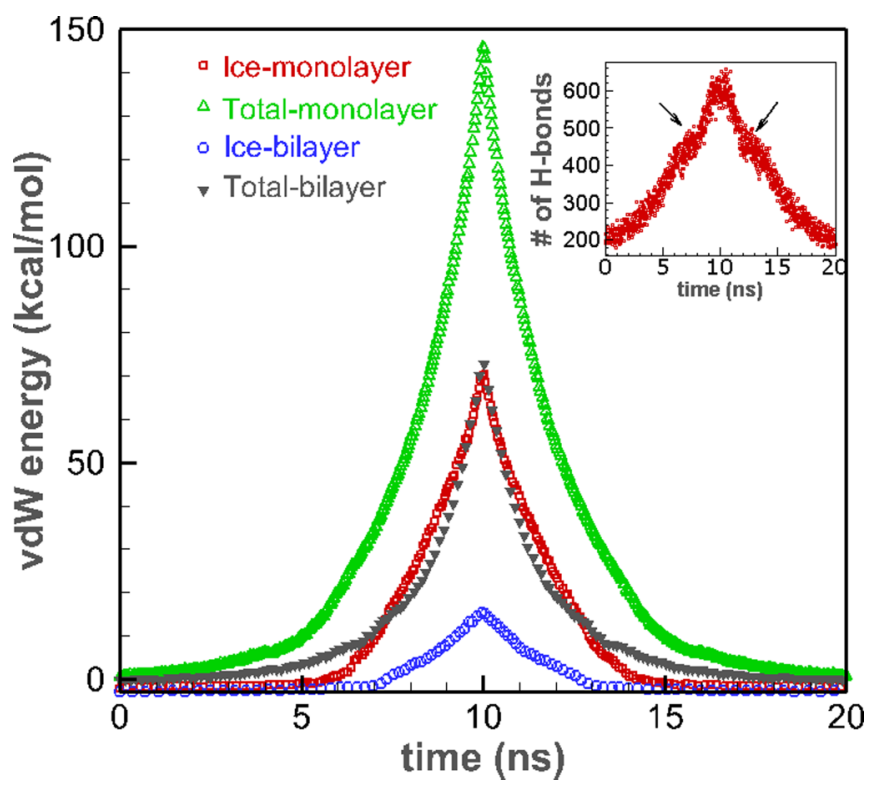

FIG. 4. Reversibility of the response of confined ice to the applied lateral pressure. The vdW energy of ice and total vdW energy as a function of the lateral pressure. The inset shows the change in the number of $\mathrm{H}$ bonds during compression (until $1 \mathrm{~ns}$ ) and expansion (beyond $1 \mathrm{~ns}$ ). Here $\mathrm{H}=6,9 \AA$ for monolayer (bilayer) to bilayer (trilayer) transitions, respectively. 


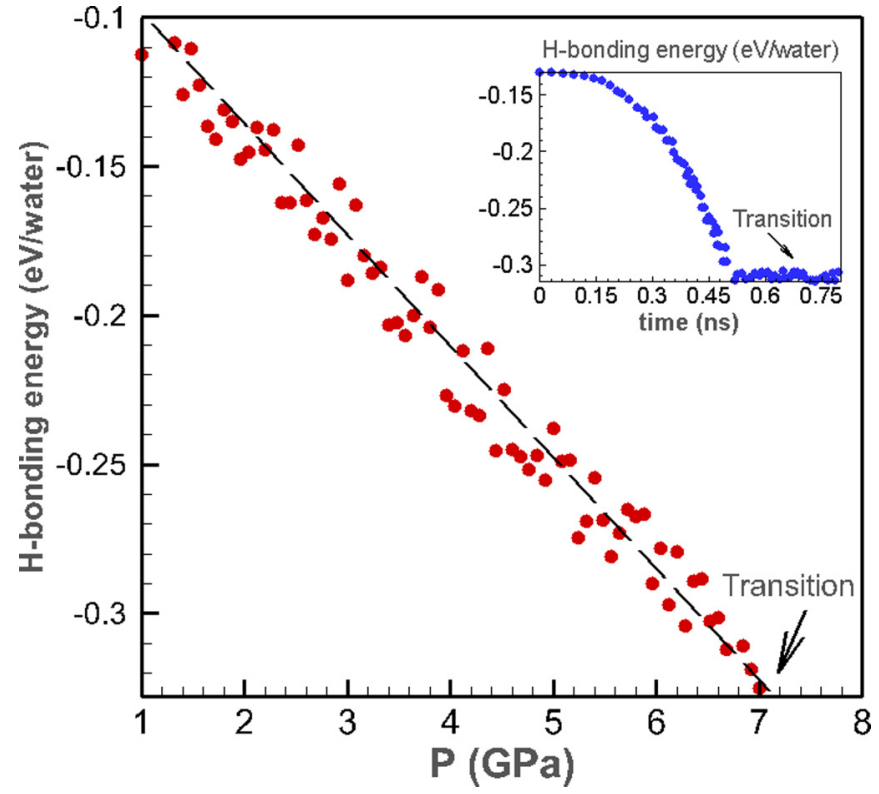

FIG. 5. The H-bonding energy per water molecule as a function of pressure using ReaxFF potentials. The inset indicates the H-bonding energy with time during compression.

monolayer to bilayer transition, we set $\mathrm{H}=6,7 \AA$ ) by moving the lateral walls with velocity of $0.0036 \AA$ ps such that the system stays in quasiequilibrium. In both cases, after a few ns of compression, bilayer (monolayer) ice is transformed into trilayer (bilayer) ice with $\mathrm{H}=8,9 \AA(\mathrm{H}=6,7 \AA)$. By compressing the initial layers further, denser structures are formed (see below).

In Fig. 2(a), we show the variation of the pressure with MD simulation time for the transformation of monolayer ice to bilayer ice (for $\mathrm{H}=6,7 \AA$ ) and bilayer ice to trilayer ice (for $\mathrm{H}=8,9 \AA$ ). It is seen that the denser water transits in smaller pressures.

In Fig. 2(b), we show the variation of the corresponding different energy contributions (vdW and Coulomb, and the sum of them) when $\mathrm{H}=6,9 \AA$ with simulation time. As we see in Fig. 2(a), e.g., around $P \approx 19 \mathrm{GPa}$ (7 GPa), bilayer (trilayer) ice with quasi $\mathrm{AB}$-stacking structure appears (ABC stacking) — not square lattice, but rather rhombic lattice-in agreement with the MD results reported by Algara-Siller et al. [2].

The obtained high lateral pressures are due to the available space and possible stacking of formed ice for a given $\mathrm{H}$ distance. For instance, it is well known that a monolayer ice is formed within a channel with $\mathrm{H}=6.5 \AA$ [9], hence $\mathrm{H}=6.0 \AA$ is small for monolayer ice formation, and surely, much smaller for bilayer ice. Therefore, a very large pressure of about $19 \mathrm{GPa}$ is needed for transition from monolayer to bilayer. Consequently, for $\mathrm{H}=7 \AA$ the transition pressure decreases to $6 \mathrm{GPa}$. For $\mathrm{H}=8,9 \AA$ in the ordinary conditions bilayer ice is formed with $\mathrm{AB}$ stacking [2,9]. By applying large pressures instead of obtaining $\mathrm{ABA}$ stacking we found $\mathrm{ABC}$ stacking leading to the transition pressures in about $6,9 \mathrm{GPa}$ for $\mathrm{H}=9 \AA$ and $8 \AA$ respectively (see discussion below).
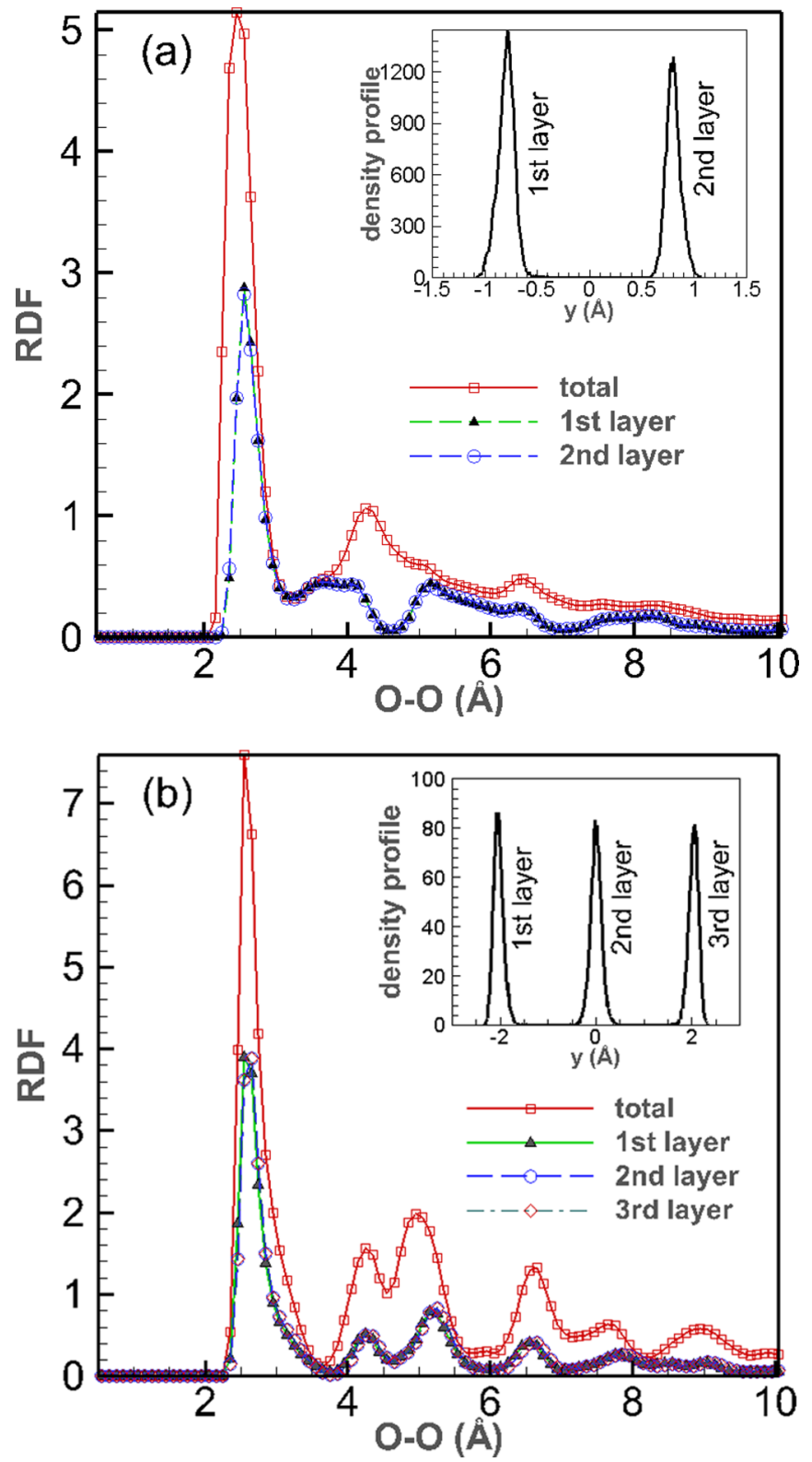

FIG. 6. The radial distribution function of the O-O distance, (a) in each of the layers in bilayer ice at $T=298 \mathrm{~K}$ and (b) trilayer ice. In each panel, the insets indicate the $y$-axis density profile. Here $\mathrm{H}=6,9 \AA$ for monolayer (bilayer) to bilayer (trilayer) transitions, respectively. The insets show the density profiles perpendicular to the layers.

In most of the cases (except for $\mathrm{H}=7 \AA$ ), the transition occurs where the curvature of the curves change in Figs. 2(a) and 2(b). Beyond this critical point, we observe a more rapid variation in all of the energy terms. However, note that because we compress the system by external walls, in our study a larger lateral pressure is used for creating layered ice as compared to the work of Algara-Siller et al. [2]. It is interesting to note that the total energy and $\mathrm{vdW}$ energy increase with increasing pressure, while the Coulomb energy decreases when the system becomes denser. The latter indicates that the $\mathrm{H}$ bonds become stronger with increasing pressure. In Figs. 3(a) and 3(b), we show snapshots of our MD results for 


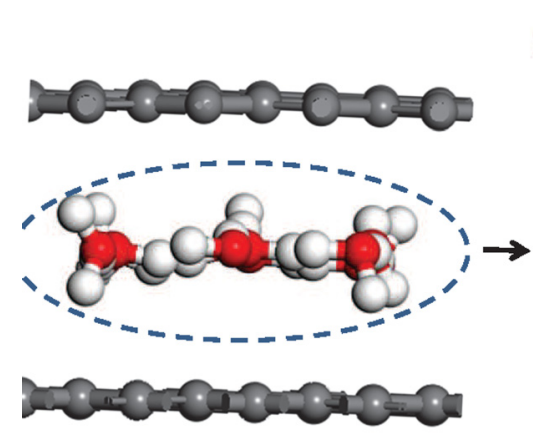

(a)

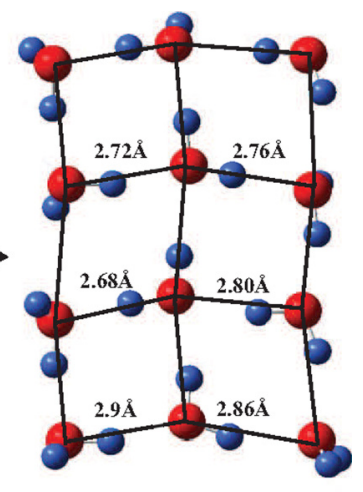

(b)

FIG. 7. (a) The optimized structure of 12 water molecules between two graphene layers and (b) the corresponding structure of single-layer water.

bilayer ice formed at $T=298 \mathrm{~K}$ under a lateral pressure of $P=19 \mathrm{GPa}$ for $\mathrm{H}=6 \AA$ A. In Figs. 3(c) and 3(d), MD results are shown for trilayer ice formed at $T=298 \mathrm{~K}$ under a lateral pressure of $P=7 \mathrm{GPa}$ for $\mathrm{H}=9 \AA$.

We also checked the reversibility of the transition process and found that the formation of multilayers is a reversible process by decreasing the pressure back to its initial value. The energy profiles vary symmetrically when we decrease the pressure for $\mathrm{H}=6,9 \AA$ (see Fig. 4). These simulations were done by moving the walls back to their initial position (expanding the system). In the inset of Fig. 4, we show that the number of $\mathrm{H}$ bonds increases with time during the compression (until $10 \mathrm{~ns}$ ) and decreases during expansion (beyond $10 \mathrm{~ns}$ ). In both cases, the transition shows up as two shoulders showed by arrows. Therefore, the transition from monolayer ice (bilayer ice) to bilayer ice (trilayer ice) is a reversible process. The $\mathrm{vdW}$ energy of ice and the total vdW energy are shown in Fig. 4. It is seen that either in the compression stage or in the expansion stage, the vdW energy of ice is lower than the total $\mathrm{vdW}$ energy beyond the transition point, which is a consequence of the smaller $\mathrm{O}-\mathrm{C}$ distance such that the strong repulsive zone of the vdW energy profile is reached.

In order to find the energy contribution from the hydrogen bonds, we performed MD simulations using reactive force-field (ReaxFF) potentials [12]. This method takes into account the charge polarization within each molecule, using electronegativity and hardness parameters based on the electronegativity equalization and charge equilibration $(\mathrm{QEq})$ methods. The variation of the total energy with pressure calculated using the ReaxFF potential is shown in Fig. 5. In the inset of Fig. 5, the H-bonding energy is shown as a function of simulation time. Here, the measurement is started at an initial configuration of bilayer ice and the pressure is slowly $(0.05 \AA / \mathrm{ps})$ increased until trilayer ice is formed at 0.5 ns. Our starting configuration has a H-bonding energy of $-0.11 \mathrm{eV} /$ atom. We find that trilayered ice has a H-bonding energy per water molecule equal to $-0.33 \pm 0.002 \mathrm{eV}$ (or $-28.0 \mathrm{~kJ} \mathrm{~mol}^{-1}$ ), which is in the upper range when comparing to the H-bonding energy of ordinary (hexagonal) ice [19], i.e., $-13-32 \mathrm{~kJ} \mathrm{~mol}^{-1}$. When gradually applying pressure,

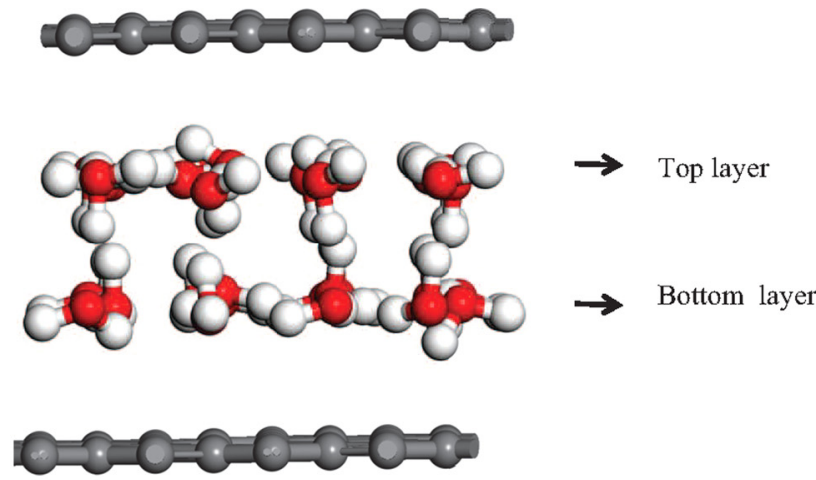

(a) (b)

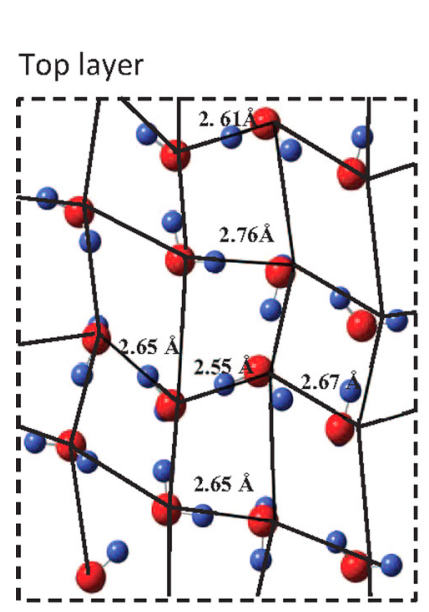

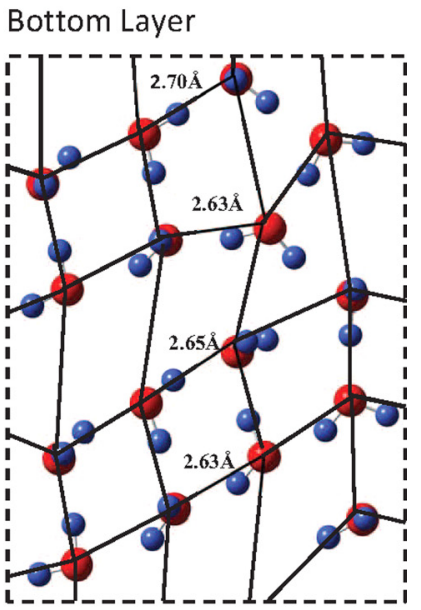

(c)
FIG. 8. (a) The optimized structure of 32 water molecules between two graphene layers and (b),(c) the corresponding structure of each layer of formed bilayer water.

we find that the absolute value of the H-bonding energy gradually increases, which is a result of the decrease in the distance between $\mathrm{O}-\mathrm{H}-\mathrm{O}-\mathrm{H}-\mathrm{O}$ bonds. By increasing the pressure, the $\mathrm{H}$ bonding increases, which is due to smaller $\mathrm{H}$-bonding distance and stronger Coulomb interaction between neighbor water molecules. Increasing the H-bond energy with increasing pressure makes the water denser, i.e., the density of confined water can be higher than $1 \mathrm{~g} / \mathrm{cm}^{3}$. The latter causes a complex rearrangement of the H-bond network and unexpected water flow, slip length, and viscosity of confined water $[10,24,25]$.

We turn back to the TIP4P results. The radial distribution function (RDF) of formed bilayer ice is (for $\mathrm{H}=6 \AA$ ) shown in Fig. 6(a) which indicates that the O-O distances are around $2.55 \AA$. In Fig. 6(b), we show the RDFs of the trilayer ice and the total RDF (for $\mathrm{H}=9 \AA$ ). The O-O distance in trilayer ice is about $2.45 \AA$ and the distance between the ice layers is found to be $2 \AA$ [see insets of Figs. 6(a) and 6(b)]. The insets in each panel shows the density profile perpendicular to the layers indicating the well defined layering of the water molecules. The distance between layers of trilayer (bilayer) found to be $2 \AA(1.4 \AA)$ which explain the large lateral 


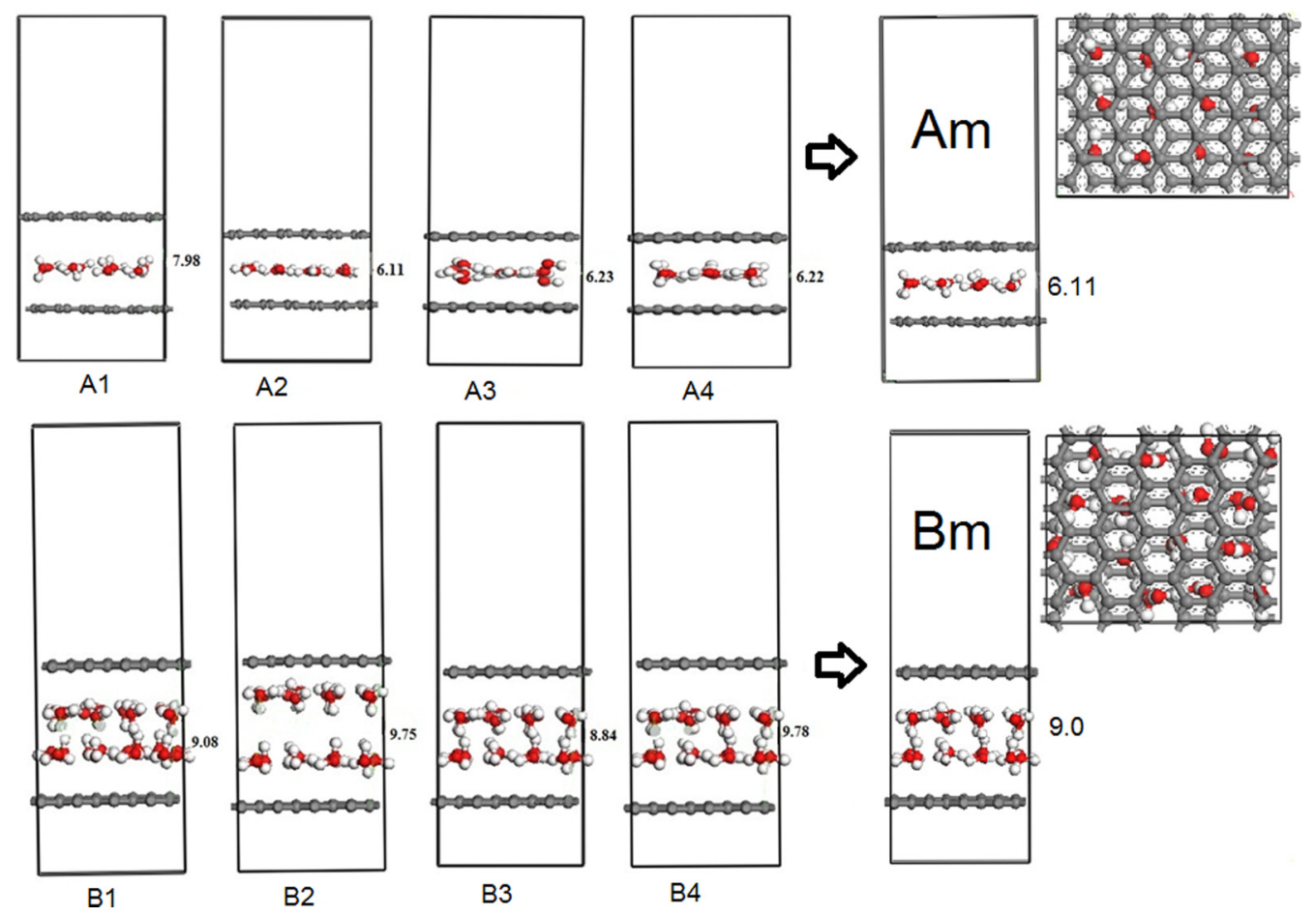

FIG. 9. Four different initial configurations for monolayer (A1,A2,A3,A4) and bilayer (B1,B2,B3,B4) ice of 12 and 32 water molecules confined between graphene layers, and the top and side views of the corresponding final optimized structures Am and Bm (shown in Figs. 7 and 8).

pressure for transiting from bilayer (monolayer) to trilayer (bilayer).

It is worthwhile to mention that we performed several additional MD simulations with different initial configurations (using TIP4P) for monolayer ice confined between two graphene layers which are separated by $\mathrm{H}=6 \AA$. By increasing the pressure, we found the monolayer to bilayer ice transition (the results are not presented here). We also changed the initial randomness of the simulation which creates many different initial random velocities and repeated the simulations and found the same results. That is because when we equilibrate the system, all of the water molecules arrange themselves inside the capillary in the same way and form monolayer or bilayer ice, depending on the $\mathrm{H}$ value. The reversibility of the results is independent of the initial random velocity. We found that the high pressure increases the coordination number of water molecules.

\section{B. Ab initio results}

In order to investigate the density effects on the O-O distance, we performed two sets of $a b$ initio calculations. In the first (second) case, we added 12 (32) water molecules in our computational unit cell between two graphene layers and applied periodic boundary conditions in the $x-y$ plane. In Figs. 7 and 8, we show the optimized structures for the two examined systems. The graphene-graphene distance is found to be 6.11 and $9.0 \AA$, respectively. The final optimized structures indicate that the density of water in the first system is around $2.0 \mathrm{~g} \mathrm{~cm}^{-3}$ and in the second one is $2.1 \mathrm{~g} \mathrm{~cm}^{-3}$. The O-O distance in both cases is shown in Figs. 7(b), 8(b), and $8(\mathrm{c})$, respectively. It is seen that in the first system12 water molecules-the $\mathrm{O}-\mathrm{O}$ distance varies between $2.7-$ $2.9 \AA$, which is in agreement with previous water simulation results [2,9]. However, for the higher density case, with 32 water molecules, the shorter $\mathrm{O}-\mathrm{O}$ radial distance is found to be around $2.6 \AA$; see Figs. 8(b) and 8(c). In another $a b$ initio calculation, we found that by increasing the density of water, the O-O distance can be shorter, e.g., $2.5 \AA$ found for $2.2 \mathrm{~g} \mathrm{~cm}^{-3}$ (the results are not shown here). It is interesting to note that our $a b$ initio results for the lattice structure of monolayer ice-shown in Fig. 7-are in agreement with the ReaxFF results [9]. The results are also consistent with recently published $a b$ initio results [26,27], while the recently published papers did not report the effects of density on the $\mathrm{O}-\mathrm{O}$ distance change. We emphasize that $a b$ initio calculations for ice subjected to high pressure require more studies, which is beyond the scope of this work.

Finally in order to test the independency of the results to the initial configurations in $a b$ initio calculations, we repeated all of the above-mentioned calculations by considering four different initial configurations with different geometries for the monolayer (named A1, A2, A3, A4) and bilayer (named B1, B2, B3, B4) ice between bilayer graphene, as shown in Fig. 9. We obtained almost the same final optimized structure (Am and $\mathrm{Bm}$ ) after optimization (full relaxation) for all structures (for monolayer ice and bilayer ice). Therefore, the DFT relaxations from different starting points are independent of the initial starting configurations. The distances between graphene layers in $\mathrm{Am}$ and $\mathrm{Bm}$ were found to be 6.11 and $9.0 \AA$, respectively. 


\section{DISCUSSION AND CONCLUSIONS}

In this study, we did not focus on the details of the atomic structure of bilayer and trilayer ice (which was already studied extensively by various groups). Since the publication of the ice square study of Algara-Siller et al. [2], many studies have been done on confined water under high pressures (see [9,26-28] and reference therein). However, the observation of square ice was challenged later [7]. Most of the theoretical papers studied the structural properties of confined water under high compressions and proposed a phase diagram of the confined water. For instance, Corsetti et al. [27] studied the phase diagram of nanoconfined monolayer ice (with possible square and hexagonal network) using DFT with a nonlocal vdW exchange-correlation functional. They also found that while DFT and other force-field models are able to produce semisquare monolayer ice, obtaining the square bilayer using both DFT and TIP4P(2005) is doubtful and was found to be unstable with respect to the high-density phases [26]. The latter is also in agreement with our previous findings [9].

Here we do not focus on the structure, instead we find the irreversibility in the compression-expansion processes as well as shorter $\mathrm{O}-\mathrm{O}$ distances in highly condensed ice. From the technical point of view, the structural transitions depend on the parameters of the force fields, especially vdW parameters which control the distance of ice and the graphene layers. We employed one of the dependable water models [TIP4P(2005)] which has already been validated for different phases of ice [11]. Our results for shorter O-O distance and stronger $\mathrm{H}$ bonding under high pressure are in agreement with those reported by Zhang et al. [29], where they investigated structural changes, phase diagram, and vibrational properties of hydrogen hydrate in the filled-ice phase (for pressures up to $60 \mathrm{GPa}$ and O-O distances about $2.44 \AA$ ) $C_{2}$ by using a first-principles molecular dynamics simulation. The structure of compressed ice, as shown in Figs. 3(b) and 3(d) in our study, is rhombic lattices with $\mathrm{AB}$ stacking for bilayer and $\mathrm{ABC}$ stacking for trilayer, which are in agreement with previous results [2].

Moreover, in this work, we observed an ABC-hexagonal trilayer ice structure; although previous work [30] has extensively investigated trilayer structures, this particular conformation was not observed. The high compression that we achieved in this work has allowed us to observe an $\mathrm{ABC}$ structure rather than an ABA structure [30]. Despite the difference in stacking, the hexagonal structure is still seen in both stacking configurations. The AB stacking that we predicted for a bilayer has been witnessed previously [31], but for larger separations than the 7 and $6 \AA$ used here. As this system has been compressed much more than previous simulations, we managed to observe an O-O distance of $2.45 \AA$ from a classical molecular dynamics force field. Although we do not observe the square ice structure obtained by Algara-Siller et al. [2], we have observed different stacking configurations and much smaller O-O separations than previously.

In the presence of a high lateral external pressure, especially when water is confined between two graphene layers, the absolute values of the vdW, Coulomb energy, and number of hydrogen bonds become larger. We found that there is a threshold in the lateral pressure beyond which the $\mathrm{O}-\mathrm{O}$ distance is no longer equal to $2.8 \AA$, but instead is $2.5 \AA$, and water becomes denser. Consequently, a reversible transition from monolayer (bilayer) ice to bilayer (trilayer) ice occurs. We showed that by increasing the lateral pressure, the lattice constant shortens for confinement width $\mathrm{H}=9 \AA$ for trilayer ice. This is promising because by fixing the confinement size and tuning the lateral pressure, we can control the interlayer distance (and number of layers) between the ice layers. We further justified the short $\mathrm{O}-\mathrm{O}$ distance by performing $a b$ initio calculations of two high densities of water encapsulated by graphene.

Finally, we discuss the possibility of a shorter O-O distance from both an experimental and theoretical point of view. There are approximately 16 different ice structures that have been confirmed using various techniques that include neutron scattering, infrared spectroscopy, and Raman spectroscopy [32]. At a temperature of $300 \mathrm{~K}$ and with pressures of $>1 \mathrm{GPa}$, there are three potential bulk water structures, VI, VII, and X. Assuming that confinement does not change the fundamental physics of the water molecules, the trend and O-O separations should be similar between bulk and 2D confined systems. Under very high pressures-where we are most likely to expect our model to break down-we should look at the structures in the VII and $\mathrm{X}$ cases. At low pressures, a $\mathrm{H}$ atom is localized (or bonded) more to one $\mathrm{O}$ atom than to the other, so there is an asymmetric potential when $\mathrm{H}$ sits between two $\mathrm{O}$ atoms. At higher pressures, in the VII structure, the $\mathrm{H}$ atom sits closer to one $\mathrm{O}$ atom than the other, but there is a symmetric potential between the two $\mathrm{O}$ atoms so it is possible for the $\mathrm{H}$ atom to hop between the two $\mathrm{O}$ atoms [33]. In this structure, the separation between the $\mathrm{O}$ atoms is $2.4 \AA$ [33-35], based on ab initio calculations [33] and infrared experiments [35]. When the VII structure is compressed further, the $\mathrm{H}$ atom potential between two $\mathrm{O}$ atoms is perfectly symmetric with one potential well; the $\mathrm{O}-\mathrm{O}$ distance is then expected to be compressed down to $2 r_{O-H}$, where $r_{O-H}$ is the $\mathrm{O}-\mathrm{H}$ bond distance [33]. It may be possible to get an O-O distance less than $2.5 \AA$, and arguably lower separations are possible in confined water, which have not been confirmed yet experimentally. We conclude that the shorter $\mathrm{O}-\mathrm{O}$ distance is possible, as we found in our simulations.

\section{ACKNOWLEDGMENTS}

This work was supported by the Flemish Science Foundation (FWO-Vl) and the Methusalem Foundation.
[1] K. S. Novoselov, A. K. Geim, S. V. Morozov, D. Jiang, Y. Zhang, S. V. Dubonos, I. V. Grigorieva, and A. A. Firsov, Science 306, 666 (2004).

[2] G. Algara-Siller, O. Lehtinen, F. C. Wang, R. R. Nair, U. Kaiser, H. A. Wu, A. K. Geim, and I. V. Grigorieva, Nature (London) 519, 443 (2015).
[3] T. Bjorkman, A. Gulans, A. V. Krasheninnikov, and R. M. Nieminen, Phys. Rev. Lett. 108, 235502 (2012).

[4] S. P. Koenig, N. G. Boddeti, M. L. Dunn, and J. S. Bunch, Nat. Nanotechnol. 6, 543 (2011).

[5] R. Zangi and A. E. Mark, Phys. Rev. Lett. 91, 025502 (2003). 
[6] Gregory R. Medders, Andreas W. Gotz, Miguel A. Morales, P. Bajaj, and Francesco Paesani, J. Chem. Phys. 143, 104102 (2015).

[7] W. Zhou, K. Yin, C. Wang, Y. Zhang, T. Xu, A. Borisevich, L. Sun, J. C. Idrobo, M. F. Chisholm, S. T. Pantelides, R. F. Kliem, and A. R. Lupini, Nature (London) 528, E1 (2015).

[8] H. Qiu, X. C. Zeng, and W. Guo, ACS Nano 9, 9877 (2015).

[9] M. Sobrino Fernandez Mario, M. Neek-Amal, and F. M. Peeters, Phys. Rev. B 92, 245428 (2015).

[10] M. Neek-Amal, F. M. Peeters, Irina V. Grigorieva, and A. K. Geim, ACS Nano 10, 3685 (2016).

[11] J. L. Abascal and C. Vega, J. Chem. Phys. 123, 234505 (2005).

[12] A. C. T. van Duin, S. Dasgupta, F. Lorant, and W. A. Goddard, J. Phys. Chem. A 105, 9396 (2001); A. C. T. van Duin and J. S. S. Damste, Org. Geochem. 34, 515 (2003).

[13] S. Plimpton, J. Comput. Phys. 117, 1 (1995).

[14] A. Cheng and W. A. Steele, J. Chem. Phys. 92, 3858 (1990).

[15] E. Sanz, C. Vega, J. L. F. Abascal, and L. G. MacDowell, Phys. Rev. Lett. 92, 255701 (2004).

[16] G. Malenkov, J. Phys.: Condens. Matter 21, 283101 (2009).

[17] R. Zangi and A. E. Mark, J. Chem. Phys. 120, 7123 (2004).

[18] M. Sobrino Fernandez, F. M. Peeters, and M. Neek-Amal, Phys. Rev. B 94, 045436 (2016).

[19] M. Chaplin, arXiv:0706.1355.

[20] P. Giannozzi, S. Baroni, N. Bonini, M. Calandra, R. Car, C. Cavazzoni, D. Ceresoli, G. L. Chiarotti, M. Cococcioni, I. Dabo, A. Dal Corso, S. Fabris, G. Fratesi, S. de Gironcoli, R. Gebauer, U. Gerstmann, C. Gougoussis, A. Kokalj, M. Lazzeri, L. Martin-Samos, N. Marzari, F. Mauri, R. Mazzarello, S. Paolini, A. Pasquarello, L. Paulatto, C. Sbraccia, S. Scandolo, G. Sclauzero, A. P. Seitsonen, A. Smogunov, P. Umari, and R. M. Wentzcovitch, J. Phys.: Condens. Matter 21, 395502 (2009).
[21] J. P. Perdew, K. Burke, and M. Ernzerhof, Phys. Rev. Lett. 77, 3865 (1996).

[22] S. Grimme, J. Antony, S. Ehrlich, and S. Krieg, J. Chem. Phys. 132, 154104 (2010).

[23] H. J. Monkhorst and J. D. Pack, Phys. Rev. B 13, 5188 (1976).

[24] Xingcai Qin, Quanzi Yuan, Yapu Zhao, Shubao Xie, and Zhongfan Liu, Nano Lett. 11, 2173 (2011).

[25] B. Radha, A. Esfandiar, F. C. Wang, A. P. Rooney, K. Gopinadhan, A. Keerthi, A. Mishchenko, A. Janardanan, P. Blake, L. Fumagalli, M. Lozada-Hidalgo, S. Garaj, S. J. Haigh, I. V. Grigorieva, H. A. Wu, and A. K. Geim, Nature (London) 538, 222 (2016).

[26] F. Corsetti, J. Zubeltzu, and E. Artacho, Phys. Rev. Lett. 116, 085901 (2016).

[27] F. Corsetti, P. Matthews, and E. Artacho, Sci. Rep. 6, 18651 (2016).

[28] W.-H. Zhao, J. Bai, L.-F. Yuan, J. Yang, and X. C. Zeng, Chem. Sci. 5, 1757 (2014).

[29] Jingyun Zhang, Jer-Lai Kuo, and Toshiaki Iitaka, J. Chem. Phys. 137, 084505 (2012).

[30] YinBo Zhu et al., J. Phys. Chem. C 120, 8109 (2016).

[31] YinBo Zho, FengChao Wang, Jaeil Bai, Xiao Cheng Zeng, and HengAn Wu, ACS Nano 9, 12197 (2015).

[32] M. Guthrie, J. Phys.: Condens. Matter 27, 153201 (2015).

[33] L. Lin, J. A. Morrone, and R. Car, J. Stat. Phys. 145, 365 (2011).

[34] Y. Finkelstein and R. Moreh, J. Chem. Phys. 139, 044716 (2013).

[35] K. Aoki, H. Yamawaki, M. Sakashita, and H. Fujihisa, Phys. Rev. B 54, 15673 (1996). 